\title{
A Simulation Study on Defectivity in Directed Self-assembly Lithography
}

\author{
Katsuyoshi Kodera, Hideki Kanai, Hironobu Sato, Yuriko Seino, Katsutoshi Kobayashi, Yusuke Kasahara, \\ Hitoshi Kubota, Naoko Kihara, Yoshiaki Kawamonzen, Shinya Minegishi, Ken Miyagi, Masayuki Shiraishi, \\ Toshikatsu Tobana, Satoshi Nomura and Tsukasa Azuma
}

\author{
DSA Research Dept., EUVL Infrastructure Development Center, Inc., \\ 16-1 Onogawa, Tsukuba-shi, Ibaraki-ken, 305-8569, Japan \\ katsuyoshi.kodera@eidec.co.jp
}

\begin{abstract}
We have investigated the generation mechanisms of pattern defects in directed self-assembly (DSA) lithography using a simulation method based on self-consistent field theory (SCFT). The SCFT simulation results could reproduce grid defects, which are considered to be a kind of hexagonally perforated lamellar (HPL) metastable phase, as one of the characteristic pattern defects in the coordinated line epitaxy (COOL) DSA lithography process using polystyrene-block-poly(methyl methacrylate) (PS-b-PMMA). We found that the grid defects were susceptible to be generated when a neutral layer was weakly attractive to PMMA block. Investigation of the PMMA segment node density profiles in the grid defects revealed that the terminal segments of the PMMA blocks indicated higher node density immediately above the neutral layer. These simulation results imply that the generation mechanism of the grid defects is strongly related with the interaction between the neutral layer bottom wafer and the terminal segment of the PMMA block.
\end{abstract}

Keywords: directed self-assembly lithography, coordinated line epitaxy process, defectivity, grid defect, self-consistent mean field theory

\section{Introduction}

Directed self-assembly (DSA) lithography, which combines self-assembled materials and lithographically defined prepatterns, is a promising low-cost candidate for extending optical/EUV lithography beyond sub-20 nm. Many applications such as hole shrink [1-5] and line/space (L/S) patterning for FinFET device [6] have been reported in recent years. In the L/S applications, some prospective process flows such as the lift-off process [7], the LiNe process [8], the SMART ${ }^{\mathrm{TM}}$ process [9], and the coordinated line epitaxy (COOL) process [10-14] have been demonstrated in full-wafer experiments. These demonstrations manifested that defects constituted one of the most important obstacles concerning practical applications of the DSA lithography. So far, the defect densities still have a couple of orders of magnitude worse than a target of 0.01 defects $/ \mathrm{cm}^{2}$ prescribed by the International Technology Roadmap for Semiconductors (ITRS) [15].

In our recent studies [10-14], we have investigated grid defects, which are considered to be a kind of hexagonally perforated lamellar (HPL) metastable phase, as one of the characteristic pattern defects in the coordinated line epitaxy (COOL) DSA lithography process using polystyrene-block-poly(methyl methacrylate) (PSb-PMMA). Since the grid defects observed in the COOL process, as shown in Fig. 1 (a), appeared a quasi-hexagonal arrangement on the neutral layer after the PMMA block removal, they looked like the HPL phase extensively observed as a long-lived metastable phase in bulk systems $[16,17]$. Although the details of the grid defects have been intensively investigated by use of the small-angle X-ray scattering method (SAXS) [18], helium ion microscopy (HIM) [19], and conventional secondary electron microscopy (SEM) [10-14,20], the origin of the defects has yet to be clarified. In this work, we report on the generation mechanisms of the grid defects, presenting findings obtained using a simulation method based on self-consistent mean field theory (SCFT) [21]. In Chapter 2, we introduce our simulation models. In Chapter 3, we discuss our simulation results concerning the metastable morphologies including state 
of the grid defects. In Chapter 4, we discuss the stability of the grid defect in accordance with the change of the neutral layer affinities. These simulation results agreed well with our experimental results. In Chapter 5, we report on the conformation of the block copolymer chain in the grid defects and discuss the origins of them.

(a)

(b)
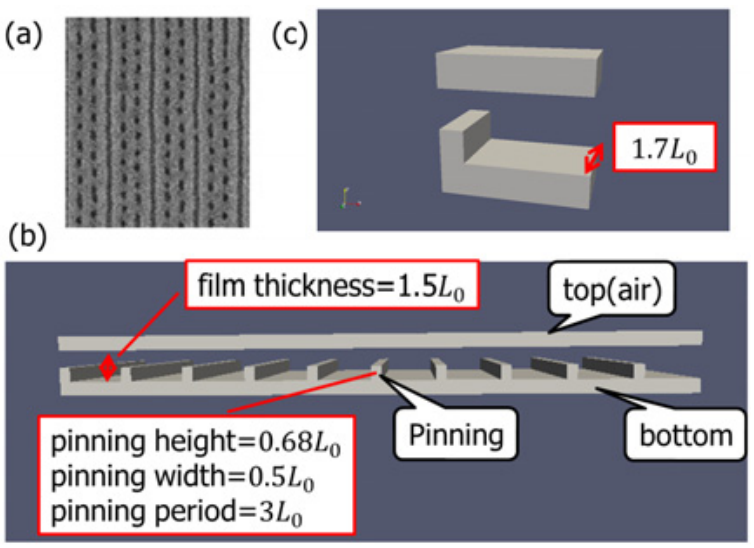

Fig. 1. (a) Grid defects observed in the COOL process. (b) and (c) Schematic view of the simulation model.

\section{Simulation models}

In this work, we have investigated the generation mechanisms of the grid defects observed in the COOL process [10-14]. We modeled the COOL process using the SCFT. Fig. 1 (b) shows a schematic view of our simulation model. We modeled symmetric $\mathrm{AB}$ diblock copolymers as a standard continuous Gaussian chain composed of segments $\mathrm{A}\left(N_{A}=10\right)$ and segments B ( $\left.N_{B}=10\right)$. In the following, PMMA segment is represented as A and PS segment is represented as B. Interactions between $\mathrm{A}$ and $\mathrm{B}$ blocks are mediated through the Flory-Huggins parameter $\chi_{A B}$. $\left(\chi_{A B} N=20\right)$ In the case of the PS- $b$-PMMA, a reference value of $\chi_{A B} N=20$ roughly corresponds to forming lamella pitch $L_{0}=30 \mathrm{~nm}$. The PS- $b$-PMMA film is filled between the top layer (air) and the bottom layer (neutral layer). The film thickness (distance between top and bottom layers) is $1.5 L_{0}$. And the stripes of the resist guides (pinning layers) are periodically arranged on the bottom layer in the COOL process. The width, height and pitch of the pinning stripes are defined $0.5 L_{0}, 0.68 L_{0}$, and $3 L_{0}$, respectively. In our SCFT simulations, we used periodic boundary condition to approximate a large system as shown in Fig. 1 (b) using a small unit cell as shown in Fig. 1 (c). System size along deep direction is defined $1.7 L_{0}$. Confinement of the BCP (top, bottom and pinning) is modeled using the masking method, first introduced by Matsen [22]. The masked top particles are neutral to two $\mathrm{AB}$ blocks $\left(\chi_{A-T O P}=2, \chi_{B-T O P}=2\right)$ and the pinning layer is attractive to PMMA block (referred to as A) $\left(\chi_{A-P I N}=1, \chi_{B-P I N}=2\right)$.

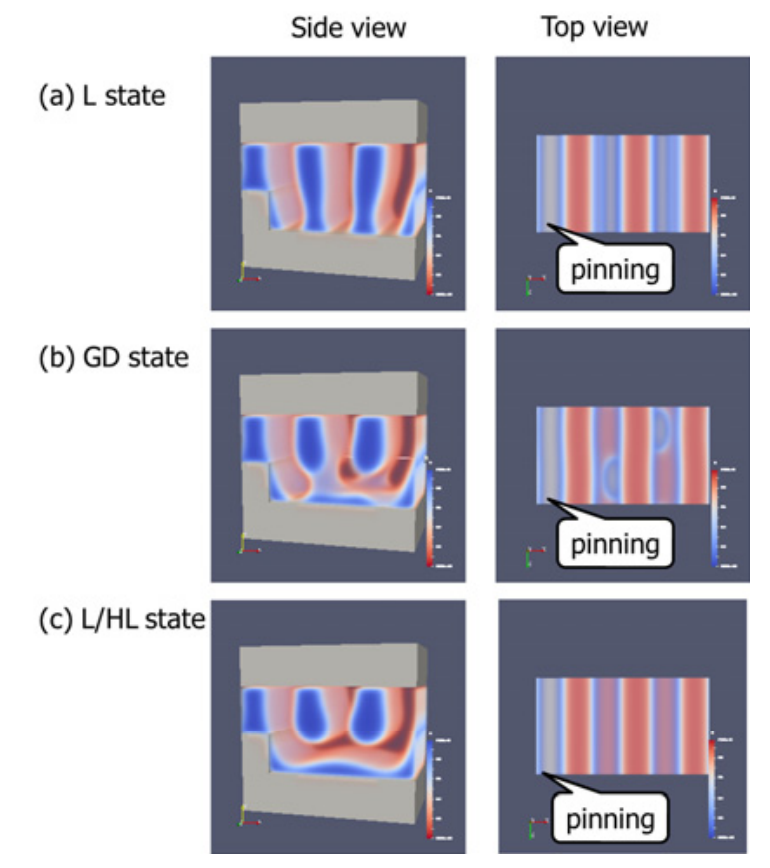

Fig. 2. SCFT simulation results. The bottom was set to be PMMA-attractive $\left(\chi_{A-T O P}=1.3, \chi_{B-T O P}=2\right)$. (a) Lamellar state. (b) Grid defect state. (c) Lamellar/Horizontal lamellar state. PMMA and PS are shown as blue and red colors, respectively.

\section{Morphology of grid defect}

As discussed in the references [10-14], the grid defects appeared differently depending on the annealing time and annealing temperature, and so their origin may be related with the metastable morphologies. Therefore, we have explored the metastable states by the free energy analysis method [23,24]. In this Chapter, we will introduce this method briefly. SCFT approximates systems with many-body interactions as non-interacting $N$ particles under effective self-consistent mean fields $\left(V_{k}(r)\right.$, where $\left.k=0,1, \cdots N-1\right)$. The segment concentration of diblock copolymers $\left(\phi_{k}(r)\right.$, where $\left.k=0,1, \cdots, N-1\right)$ can be calculated from the effective self-consistent mean fields $V_{k}(r)$. And the external self-consistent mean fields $V_{k}(r)$ are determined self-consistently from the segment concentration of diblock copolymers $\phi_{k}(r)$. Although researchers usually run self-consistent field 
simulations with random initial mean field conditions in order to obtain the most stable equilibrium state corresponding to the global minimum of free energy, we give appropriate initial conditions for the self-consistent fields and run simulations to acquire some metastable states. Because the free energy usually has some local metastable states, the calculation is trapped by one of the metastable states. By carefully giving initial conditions, we have found that we can obtain some typical metastable states and their free energies. In this work, self-consistent field simulations were executed with various initial values of the self-consistent mean fields $V_{k, \text { initial }}(r)$ as follows:

$$
V_{k, \text { initial }}(r)=\theta_{k}(r)+f_{k}(r)
$$

where $k$ denotes the species type $(k=0,1,2, \cdots, N-1)$, $\theta_{k}(r)$ is a Gaussian random noise function and $f_{k}(r)$ is a function to adjust the initial distribution of the self-consistent field. For example, when $f_{k}(r)$ is a periodic function with period $\Lambda$ along $\mathrm{x}$-axis $\left(f_{k}(x+\Lambda, y, z)=f_{k}(x, y, z)\right)$, the phase separation structure can easily converge toward lamellar structure along $\mathrm{x}$-axis as well if there exists such a metastable state in the free energy space. It should be noted that we should assume $\left|f_{k}(r)\right| \ll 1$ and a relatively large number of iterations of self-consistent field calculation in order to obtain a good result.

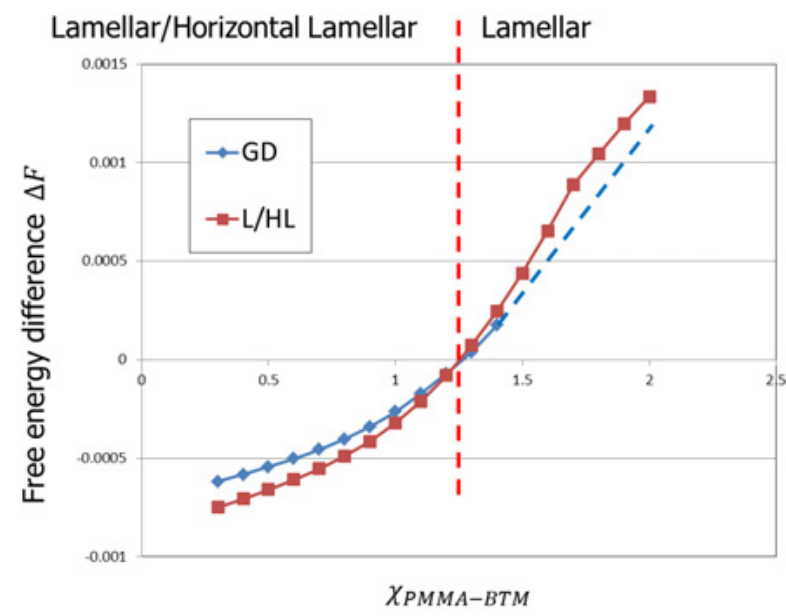

Fig. 3. The free energy differences of L/HL-state and GD-state relative to L-state with respect to $\chi_{A-T O P}$. The simulation condition $\chi_{A-T O P}=2$ corresponds to the bottom that is neutral to two blocks. And the simulation condition $\chi_{A-T O P}<2$ corresponds to the bottom that is attractive to A-component (PMMA).

The grid defects can be experimentally observed when the bottom layer is weakly attractive to PMMA. So the affinity of the bottom layer was set to be PMMA-attractive $\left(\chi_{A-T O P}=1.3, \chi_{B-T O P}=2\right)$. As shown in Fig. 2, we obtained 3 stable/metastable morphologies in this simulation condition. In this Figure, PMMA and PS are shown as blue and red colors, respectively. In this simulation condition, the vertical lamellar phase (hereinafter referred to as L-state) shown in Fig. 2 (a) was identified as the most stable state. And the grid defect state shown in Fig. 2 (b) (hereinafter referred to as GD-state) is observed as a metastable morphology. Lamellae can also orientate to a mixed lamellar phase as shown in Fig. 2 (c) (hereinafter referred to as L/HL-state) as a metastable morphology. As we can see in the right panel of Fig. 2 (b), the GD-state can be observed as quasi-hexagonal buried structures.

\section{Grid defect stability dependence on bottom affinity}

We have investigated the free energies of the 3 stable/metastable states with respect to the bottom layer affinity. In the simulation, we set $\chi_{B-T O P}$ as a fixed value $\left(\chi_{B-T O P}=2\right)$ and we altered the bottom affinity by changing the value of $\chi_{A-T O P}$. The simulation condition $\chi_{A-T O P}=2$ corresponds to the bottom that is neutral to two blocks (A and $\mathrm{B}$ ) and the condition $\chi_{A-T O P}<2$ corresponds to the bottom that is more attractive to A-component (PMMA) than to B-component (PS). Fig. 3 shows the free energy differences of L/HL-state and GD-state relative to L-state with respect to the change of $\chi_{A-T O P}$. In almost neutral bottom conditions $\left(\chi_{A-T O P}>1.5\right)$, GD-state became unstable and we could not acquire the free energy of the GD-state. (The dashed line in this region was extrapolated.) As shown in this Figure, when the bottom is attractive to PMMA $\left(\chi_{A-T O P}<1.2\right), \mathrm{L} / \mathrm{HL}$-state was calculated as the most stable state and GD-state and L-state are metastable. On the other hand, when the bottom is almost neutral to two blocks $\left(\chi_{A-T O P}>1.3\right)$, L-state becomes the most stable state and GD-state and L/HL-state becomes metastable states. As the bottom becomes more attractive to PMMA, the free energy difference between L-state and GD-state becomes smaller. This simulation result suggests that the GD-state becomes more stable as the bottom layer is more attractive to PMMA. This simulation result is consistent with the experimental behavior that manifests that the GD-state can be frequently observed when the bottom layer is PMMA-attractive [10-14]. And moreover, our simulation results suggest that the L-state is in thermal equilibrium 
and GD-state is metastable in almost neutral bottom layer conditions. The results suggest that the grid defects can be statistically eliminated by changing the annealing conditions (for example, long annealing).

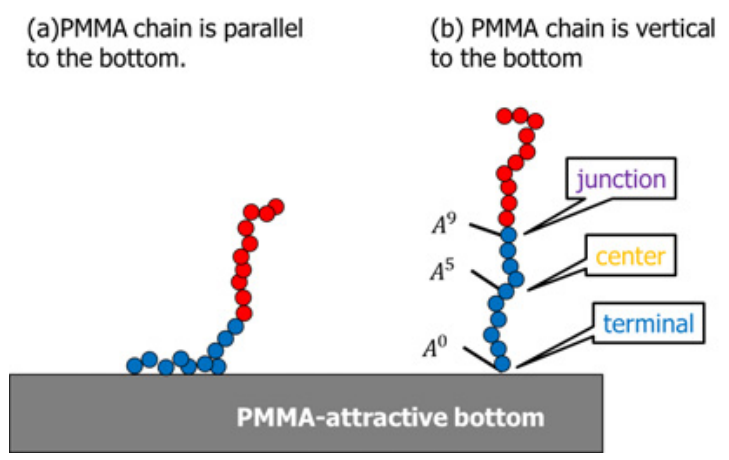

Fig. 4. Schematic view of BCP chain conformations on PMMA-attractive bottom. (a) PMMA chain is nearly parallel to the bottom layer. (b) PMMA chain is almost vertical to the bottom layer.

\section{Polymer morphologies of the grid defects}

In order to understand more about the GD-state, we have investigated the conformation of the BCP chains. The conformation of the polymer chain is an important factor that determines the macroscopic morphology of phase separation. Fig. 4 shows a schematic view of $\mathrm{AB}$ diblock copolymer $A_{10} B_{10}\left(A^{0} A^{1} \cdots A^{9} B^{10} \cdots B^{19}\right)$ on PMMA-attractive bottom layer. Blue and red particles indicate PMMA and PS, respectively. There are two possibilities of the BCP chain conformations. PMMA block chain can be nearly parallel (as shown in (a)) or vertical (as shown in (b)) to the bottom layer.

In order to investigate the conformation of the polymer chain immediately above the bottom layer, we have investigated the density distribution of each element, or "segment" of the PMMA block chain. We investigated the segment density of $A^{0}$ (the terminal segment of PMMA block), $A^{5}$ (the center segment of PMMA block) and $A^{9}$ (junction part). The segment densities of $A^{0}, A^{5}$ and $A^{9}$ are referred to as $n_{0}, n_{5}$ and $n_{9}$, respectively. And the total density of PMMA is referred to as $n_{A}$ hereinafter $\left(n_{A}=\sum_{i=0}^{9} n_{i}\right)$. When the PMMA block chain is nearly perpendicular to the bottom wall as shown in (b), $n_{0}$ should become larger than both $n_{5}$ and $n_{9}$ in the region immediately above the bottom layer. On the other hand, when the PMMA block chain is nearly parallel to the bottom layer as shown in (a), $n_{0}$ should be almost the same as $n_{5}$ in the region immediately above the bottom layer. Hence we can acquire the information of the polymer chain conformation by evaluating $n_{0}, n_{5}$ and $n_{9}$.

(a)

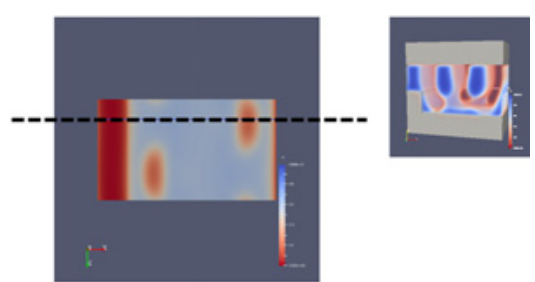

(b)

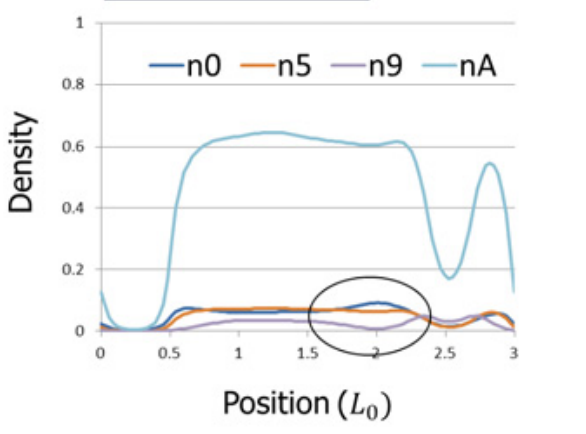

Fig. 5. (a) PMMA density distribution immediately above the bottom surface in GD-state. (b) The density profiles along the dashed line shown in (a).

Fig. 5-7 show the simulation results in the case of PMMA-attractive bottom layer $\left(\chi_{A-T O P}=1.3, \chi_{B-T O P}=2\right)$. Fig. 5 (a) shows the density distribution of PMMA block $n_{A}$ immediately above the bottom surface in GD-state. (The distance from the bottom surface is $0.014 L_{0}$.) Blue (red) region shows where the PMMA density is large (small). Fig. 5 (b) shows the PMMA segment density profiles along the dashed line shown in Fig. 5 (a). Whereas $n_{0}$ is almost the same as $n_{5}$ in most areas, it is interesting that $n_{0}$ is remarkably larger than both $n_{5}$ and $n_{9}$ in the encircled region. The encircled region was found to be located where the vertical lamellar pattern is partially connected toward the bottom. This means that PMMA blocks are nearly perpendicular to the bottom in these connected regions. On the other hand, PMMA blocks in the other regions were found to be nearly parallel to the bottom wall.

Fig. 6 and Fig. 7 show the simulation results for L/HL-state and L-state, respectively. (a) shows the density distribution of PMMA block immediately above the bottom surface. (The distance from the bottom surface is $0.014 L_{0}$.) (b) shows the PMMA density profiles along the dashed line shown in (a). In the case of both L/HL-state and L-state, $n_{0}$ is almost the same as $n_{5}$ in all regions. This means that most of the PMMA blocks 
immediately above the bottom layer are almost parallel to the bottom layer.

(a)

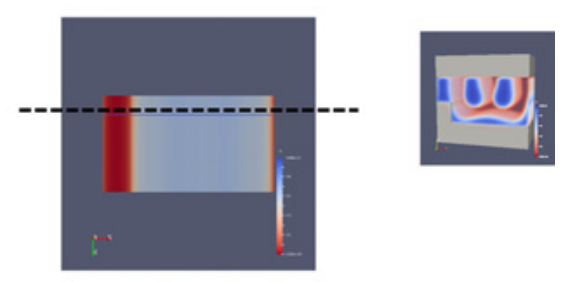

(b)

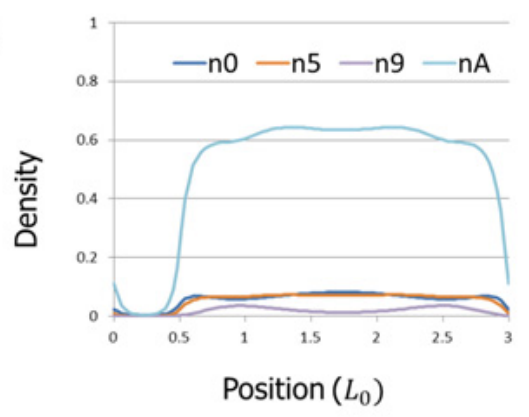

Fig. 6. (a) PMMA density distribution immediately above the bottom surface in L/HL-state. (b) The density profiles along the dashed line shown in (a).

(a)
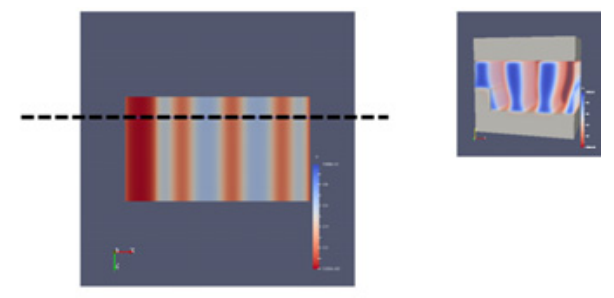

(b)

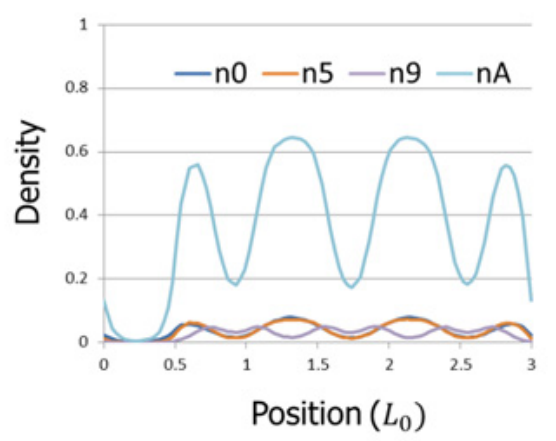

Fig. 7. (a) PMMA density distribution immediately above the bottom surface in L-state. (b) The density profiles along the dashed line shown in (a).

These characteristic conformations of PMMA block chains in GD-state can be understood as follows. In terms of the conformation entropy, polymer chains prefer to be vertical to the bottom layer, whereas the polymer chains prefer to be parallel to the bottom layer from the viewpoint of the surface free energy $F_{\text {surface }}$. Although $F_{\text {conformation }}$ is assumed to be independent of the bottom affinity, $F_{\text {surface }}$ strongly depends on the bottom affinity. Therefore, in the case of strongly PMMA-attractive bottom layer, all the PMMA blocks become parallel to the bottom layer owing to the large $F_{\text {surface }}$ energy term. On the other hand, when the bottom layer is weakly attractive to PMMA and the free energy of horizontally oriented PMMA blocks is almost equivalent to the free energy of the vertically oriented PMMA blocks, PMMA blocks can be partially parallel and partially vertical to the bottom layer simultaneously. Consequently, the two types of chain conformations can be observed in GD-state.

\section{Conclusions}

We have investigated the generation mechanisms of the grid defects observed in the COOL process using SCFT. When the neutral layer bottom is attractive to PMMA, we have found that the terminal segment of the PMMA block immediately above the bottom is locally concentrated in a certain area in GD-state. This result suggests that PMMA chains are nearly vertical to the bottom layer in this specified region. This result can be understood as follows. In GD-state, the free energy of the horizontally oriented PMMA blocks is almost equivalent to the free energy of the vertically oriented PMMA blocks, and therefore the two types of chain conformations can be observed in GD-state. Consequently, PMMA blocks immediately above the bottom in GD-state are partially parallel and partially vertical to the bottom layer simultaneously. These simulation results imply that repulsion interaction between the bottom and the terminal segment of PMMA may play an important role in the stability of the grid defects.

\section{Acknowledgments}

We used OCTA (<http://octa.jp $>)$ software in order to acquire some of the simulation results. This work was partly funded by the New Energy and Industrial Technology Development Organization (NEDO) under the EIDEC project.

\section{References}

1. Y. Seino, H. Yonemitsu, H. Sato, M. Kanno, H. Kato, K. Kobayashi, A. Kawanishi,T. Azuma, M. Muramatsu, S. Nagahara, T. Kitano, T. Toshima, $J$. Micro/Nanolithogr. MEMS MOEMS, 12 (3) (2013) 031305.

2. H. Kato, Y. Seino, H. Yonemitsu, H. Sato, M. Kanno, K. Koyayashi, A. Kawanishi,T. Imamura, M. Omura, 
N. Nakamura, T. Azuma, J. Photopolym. Sci. Technol., 26(1) (2013) 21-26.

3. H. Kato, Y. Seino, H. Yonemitsu, H. Sato, M. Kanno, K. Koyayashi, A. Kawanishi,T. Imamura, M. Omura, N. Nakamura, T. Azuma, Microelecrton. Eng., 110 (2013) 152-155.

4. K. Kodera, S. Maeda, S. Tanaka, S. Mimotogi, Y. Seino, H. Yonemitsu, H. Sato, T. Azuma, Proc. SPIE, 8680 (2013) 868015.

5. H. Sato, H. Yonemitsu, Y. Seino, H. Kato, M. Kanno, K. Kobayashi, A. Kawanishi, K. Kodera, T. Azuma, Proc. SPIE, 8680 (2013) 86801K.

6. J. Cheng, D. Sanders, H. Truong, S. Harrer, A. Friz, S. Holmes, M. Colburn, W. Hinsberg, ACS Nano, 4 (8) (2010) 4815-4823.

7. C. Liu, C. Estrada-Raygoza, H. He, M. Cicoria, V. Rastogi, N. Mohanty, H. Tsai, A. Schepis, K. Lai, R. Chao, D. Liu, M. Guillorn, J. Cantone, S. Mignot, R. Kim, J. Cheng, M. Tjio, A. Ko, D. Hetzer, M. Somervell, M. Colburn, Proc. SPIE, 9049 (2014) 904909.

8. R. Gronheid, Y. Lee, Y. Cao, Y. Her, L. D’Urzo, D. Heuvel, P. Delgadillo, A. Romo-Negreira, M. Somervell, R. Harukawa, V. Nagaswami, Proc. SPIE, 9049 (2014) 90491L.

9. J. Kim, J. Wan, S. Miyazaki, Y. Cao, Y. Her, H. Wu, K. Kurosawa, G. Lin, J. Photopolym. Sci. Technol., 26 (5) (2013) 573-579.

10. Y. Seino, Y. Kasahara, H. Sato, K. Kobayashi, K. Miyagi, S. Minegishi, K. Kodera, H. Kanai, T. Tobana, N. Kihara, T. Fujiwara, N. Hirayanagi, Y. Kawamonzen and T. Azuma, Microelectronic Engineering, 134 (2015) 27.

11. Y. Seino, Y. Kasahara, H. Sato, K. Kobayashi, H. Kubota, S. Minegishi, K. Miyagi, H. Kanai, K. Kodera, N. Kihara, Y. Kawamonzen, T. Tobana, M. Shiraishi, S. Nomura, T. Azuma, Proc. SPIE 9423 (2015) 942316.

12. Y Kasahara, Y Seino, K Kobayashi, H Kanai, H Sato, H Kubota, T Tobana, S Minegishi, K Miyagi, N Kihara, K Kodera, M Shiraishi, Y Kawamonzen, S
Nomura, T Azuma, Proc. SPIE, 9423 (2015) 94280S.

13. H. Kanai, K. Kodera, Y. Seino, H. Sato, Y. Kasahara, K. Kobayashi, K. Miyagi, S. Minegishi, N. Kihara, Y. Kawamonzen, T. Fujiwara, N. Hirayanagi, T. Tobana and T. Azuma (2015) MRS Proceedings, 1750, mrsfl4-1750-kk05-18.

14. H. Sato, Y. Seino, N. Kihara, Y. Kasahara, K. Kobayashi, K. Kodera, H. Kanai, Y. Kawamonzen, S. Minegishi, K. Miyagi, T. Tobana, N. Hirayanagi, T. Fujiwara, T. Azuma and T. Hayakawa, MRS Proceedings 1750 (2015) mrsfl4-1750-kk06-04.

15. www.itrs.net

16. Hamley, I. W. Koppi, K. A. Rosedale, J. H. Bates, F. S. Almdal, K. Mortensen, K. Macromolecules, 26 (1993) 5959.

17. Thomas, E. L. Anderson, D. M. Henkee, C. S. Hoffman, D. Nature 334 (998) 598.

18. Moon Jeong Park and Nitash P. Balsara, Macromolecules, 41 (2008) 3678.

19. Richard A. Farrell, Erik R. Hosler, Gerard M. Schmid, Ji Xu, Moshe E Preil, Michael J. Cicoria, David R. Helzer, Anton J. Devilliers, Proc. SPIE, 9051 (2014) 90510Z.

20. D. Borah, T. Ghoshal, M. T. Shaw, A. Chaudhari, N. Petkov, A. P. Bell, J. D. Holmes and M. A. Morris, Nanomater Nanotechnol, 4 (2014) 25.

21. For example, G. H. Fredrickson, The Equilibrium Theory of Inhomogeneous Polymers, Oxford University Press (2006).

22. Matsen, M. W., J. Chem. Phys. 106 (1997) 7781.

23. K. Kodera, H. Sato, H. Kanai, Y. Seino, N. Kihara, Y. Kasahara, K. Kobayashi, K. Miyagi, S. Minegishi, K. Yatsuda, T. Fujiwara, N. Hirayanagi, Y. Kawamonzen, T. Azuma, Proc. SPIE 9049 (2014) 904926.

24. K. Kodera, H. Kanai, H. Sato, Y. Seino, N. Kihara, Y. Kasahara, K. Kobayashi, K. Miyagi, S. Minegishi, T. Tobana, T. Fujiwara, N. Hirayanagi, Y. Kawamonzen, T. Azuma, J. Photopolym. Sci. Technol., 27 (2014) 31. 